\title{
Catalyzing the quantum leap
}

\author{
Kushal Seetharam ${ }^{1, \dagger, *}$ and Michael DeMarco ${ }^{2, \dagger}$
}

Edited by Shobhita Sundaram and Grant A. Knappe

\section{HIGHLIGHTS}

- A new generation of quantum technology is on the horizon which will enable innovation in a wide variety of scientific disciplines and industry sectors.

- The maturity of quantum information science (QIS) has reached an inflection point, with government and industry increasingly investing in the field in the hopes of realizing its potential.

- In order to effectively catalyze progress in QIS, we should build an adaptable workforce of scientists and engineers, which would endow this emerging innovation system with the resilience and flexibility it needs.

Given the growing hype and investment in quantum technology, it is important to understand its current landscape and how best to catalyze progress in QIS towards the eventual goal of societally-relevant innovation in materials, pharmaceuticals, industrial processes, cybersecurity and myriad other areas. We give a brief overview of the policy landscape of the emerging QIS innovation system. The most important requirement for steady progress in QIS is a robust workforce. Here, we focus on one key slice of workforce development: the training of adaptable quantum scientists and engineers.

Q uantum information science (QIS) is an emerging field of science and technology that seeks to harness the unique properties of quantum systems such as coherence, entanglement, and measurement [1] for applications in computing [2], sensing [3], and communication [4]. Progress in quantum computing is likely to have a dramatic impact across different industries by facilitating advances in optimization, artificial intelligence, cryptography, chemistry, and material science by dramatically speeding up certain computations that are intractable on even the most powerful classical computers $[5,6]$. For example,

\footnotetext{
${ }^{1}$ Department of Electrical Engineering and Computer Science, Massachusetts Institute of Technology, Cambridge, MA

${ }^{2}$ Department of Physics, Massachusetts Institute of Technology, Cambridge, $\mathrm{MA}$

${ }^{\dagger}$ These authors contributed equally.

*Corresponding author. Email: kis@mit.edu
}

The authors declare no conflict of interest.

(C) 2021 The Author(s) a sufficiently powerful quantum computer would help elucidate the reaction mechanisms that bacteria use to efficiently perform nitrogen-fixation, potentially enabling the production of cheap ammonium [7,8]; currently, $1-3 \%$ of world energy use is spent on an inefficient approximation to this process, with this resource use having serious environmental and economical consequences $[7,9,10]$ Quantum computers would also allow insight into materials such as high-temperature superconductors [8], which if understood, would lead to technology that makes both fusion energy and renewable sources like solar and wind much more viable. Quantum-enhanced sensors, meanwhile, would prove useful in myriad applications including precision timing and navigation as well as medical imaging and biochemical analysis [11]. Quantum communication protocols will alter the modern cybersecurity landscape by enabling secure communication and distributed computing [12].

Since its inception approximately forty years ago, QIS has flourished in academic environments. The field is now at an inflection point in maturity, with increasing industry involvement seeking to progress $R \& D$ in order to realize the innovations enabled by a new era of quantum technology. Federal governments are also increasing their investment in QIS as well attempting to establish a favorable policy landscape [13]. While the nascent foundations of a vibrant quantum ecosystem are starting to come together, policymakers must be thoughtful about how they organize the QIS innovation system so that the growing excitement and funding translates into effective technological progress and the capture of economic benefits from this emerging sector.

Many of the above technological applications become feasible once a large, fault-tolerant quantum computer (FTQC) exists. There is also reasonable scientific consensus that such a computer will eventually be built. However, even optimistic estimates place the arrival of such a machine at least 15-20 years away. Quantum-enhanced sensors are likely to achieve technical readiness earlier, with quantum communication maturing somewhere between sensing and computing. It is highly uncertain, however, what the actual timelines for quantum technologies to find practical applications are and where the earliest applications will be [14]. It is therefore crucial that the national QIS innovation ecosystem is resilient enough to tolerate and break through unexpectedly difficult engineering challenges that delay or halt certain technological directions, while simultaneously being flexible 
enough to exploit the discovery of unexpected directions and applications.

The long-term picture is complicated by the uncertainty in which physical hardware platforms are most suitable for different quantum technologies. For example, some of the current contenders for physical systems to encode the quantum bits (qubits) underlying quantum computers include ions held in electric traps, superconducting circuits [15], neutral atoms held in optical traps, and photons held in cavities. The main challenge in developing a FTQC is to balance the need for qubits to interact in order to perform an actual computation and the fact that undesirable interactions rapidly degrade the sensitive quantum information stored in the qubits. Each of the above mentioned platforms have different trade-offs in this regard, especially as one considers scaling the system to the qubit numbers necessary to build a FTQC. A fifth platform, the so-called topological qubits which make heavy use of large-scale entanglement, promises to be much more robust to the loss of quantum information, but the physical realization of these systems has proved to be difficult.

In this paper, we consider the challenges in building a capable new workforce for the American QIS ecosystem. We first give an overview of the current US QIS policy landscape and then discuss how resilience and flexibility can be cultivated by increasing the cross-pollination between universities, national labs, and industry firms which are starting to settle into complementary R\&D lanes, and relatedly by engaging in workforce development at all levels. The benefits of cross-polination explored in this work, however, are only one part of building resilience; immigration policy, equitable educational opportunities, consistent funding of research, and the work-life balance of academic career paths are a few of the many issues that should be addressed to truly build a robust QIS workforce.

\section{THE QIS INNOVATION SYSTEM}

\section{Policy landscape}

Quantum Information Science is unfolding across universities, national laboratories, and private industry. Underlying this tri-fold structure of quantum research is the federal government, which funds universities and national labs and determines research priorities. Civilian agency funding is now poised to take off under the National Quantum Initiative Act [16] (NQI Act), passed by Congress in 2018, with broad bipartisan support to stimulate and develop American QIS. Here we review the institutions created by the NQI Act and their roles.

The NQI Act established the National Quantum Initiative, a multi-agency program spanning the National Science Foundation, the Department of Energy, and the National Institute of Standards and Technology to support research and workforce development for QIS. It authorized $\$ 1.2 \mathrm{~B}$ for research spending and workforce development over five years and established mechanisms to coordinate QIS research across the federal agencies.

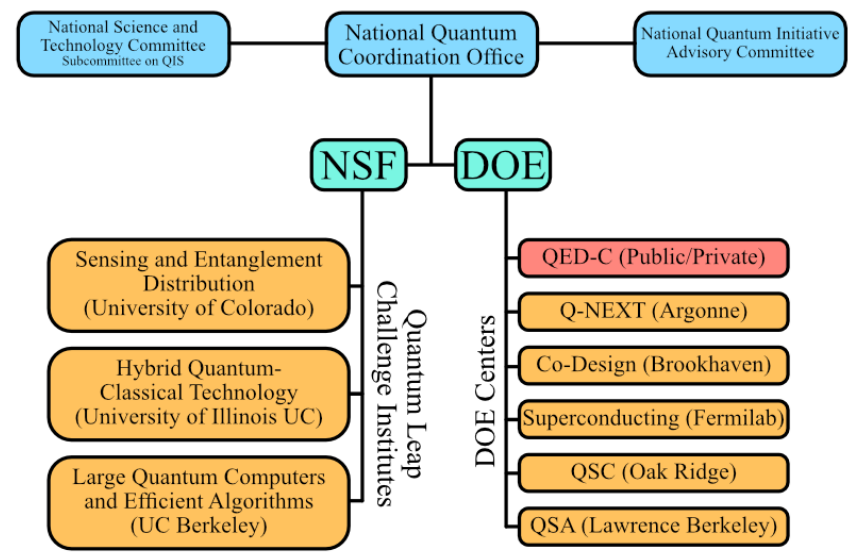

Figure 1: A selection of the governemental agencies involved in the National Quantum Initiative Act and the research initiatives they fund.

Overseeing NQI efforts is the National Quantum Coordination Office (NQCO), which serves as the point of contact for the NQI, promotes funding opportunities, conducts public outreach, and continues to set priorities [14] (See Figure 1). The NQCO director is appointed by the director of the White House Office of Science and Technology Policy. Alongside the NQCO are two coordination committees: the National Science and Technology Committee subcommittee on QIS, which coordinates QIS research budgets across federal agencies, assesses infrastructure requirements (including workforce training), and establishes priorities; and the National Quantum Initiative Advisory Committee, comprised of representatives from industry, universities, and federal labs which provides external input on the progress of the NQI and trends in QIS.

Following the passage of the NQI Act, the aforementioned National Science and Technology Committee released the National Strategic Overview for QIS in 2018 [17], addressing the actions needed to translate scientific progress in QIS into a new industry. One of their key recommendations was the development of public-private partnerships, in particular by creating a Quantum Economic Development Consortium (QED-C) which unites industry, academic, and other stakeholders with support from multiple government agencies. By identifying gaps in technology, standards, and workforce development and addressing those gaps collectively, QED-C aims to support the development of a commercial QIS industry and supply chain in the U.S.

Working alongside the QED-C are a series of innovation centers established to enhance collaboration between universities and the national laboratories. The DOE has established five new centers at major national laboratories, each with a budget of $\$ 115 \mathrm{M}$ over five years:

- Q-NEXT (Argonne) will focus on materials fabrication and testbed development

- The Co-design Center for Quantum Advantage (Brookhaven) will focus on improved material design 
for hardware and hardware-specific co-design of error-correction schemes and algorithms

- The Superconducting Quantum Materials and Systems Center (Fermilab) will focus on improving coherence and quality of quantum technology

- The Quantum Science Center (Oak Ridge) will focus on topological materials, quantum algorithms, and sensors

- The Quantum Systems Accelerator (Lawrence Berkeley) will focus on improving hardware platforms as well as co-design of algorithms targeted towards scientific discovery

Additionally, the NSF has invested $\$ 75 \mathrm{M}$ in three new Quantum Leap Challenge Institutes: one focusing on sensing and entanglement distribution, led by the University of Colorado; one focusing on hybrid quantum-classical technology led by the University of Illinois at Urbana-Champaign, and one focused on the development of large scale quantum computers and efficient algorithms led by the University of California at Berkeley.

\section{Systemic Challenges}

A serious challenge facing QIS is the aforementioned uncertainty in the trajectory of the industry: the final goal, a FTQC, is likely to be a few decades away, and the development of NISQ devices is still in progress. Many applications of NISQs have yet to be discovered; on the other hand, some of the expected applications will likely turn out to be infeasible. Uncertainty is difficult for any industry, and it will be doubly so for a developing ecosystem spread across universities, national labs, and private companies like QIS. The QIS community will need resiliency and robustness to navigate the twisting course ahead.

The NSF and DOE innovation centers illustrate an illuminating approach to this issue: developing a flexible, well trained, and multidisciplinary workforce. As research approaches succeed or fail, and as private companies rise or fall, QIS will need a workforce that can smoothly transition among universities, national laboratories, and private industry; the researchers, technicians, and engineers working in QIS will require fluency in multiple approaches to quantum computing and multiple lanes of the industry.

With this in mind, we next take a deep dive into one of the innovation centers and explore its strengths and weaknesses from a workforce development point of view.

\section{Case Study: Quantum Systems Accelerator}

The Quantum Systems Accelerator (QSA) is one of the national QIS research centers created by the DOE in accordance with the NQIA. It has been allocated $\$ 115 \mathrm{M}$ in funding over five years with a mission centered on co-designing quantum algorithms and quantum hardware platforms in order to demonstrate a quantum advantage in scientific applications. The network of members participating in the center include national labs like Lawrence Berkeley, Sandia, and MIT Lincoln Laboratory as well as universities such as Berkeley, Caltech, Duke, Harvard, MIT, University of Colorado Boulder, University of Maryland, and University of Texas Austin. The center will also engage with industry through, for example, activities involving QED-C member companies.

Research at the center will encompass the three major types of hardware platforms for programmable quantum computing: superconducting circuits, trapped ions, and neutral atoms. Projects will develop the engineering and control necessary to mitigate errors and scale these platforms, while simultaneously investigating hardware-tailored error-resistant algorithms for scientific problems which may demonstrate a practical quantum advantage compared to classical computational methods. The center also intends to engage in workforce development activities that help cultivate a future pool of trained quantum scientists, engineers, and technicians.

The QSA provides a scaffold for coordinated R\&D and workforce development efforts in QIS, and centers like it have the potential to act as a critical nexus of all three primary actors in the innovation ecosystem: universities, national labs, and industry firms. For example, the $\$ 115 \mathrm{~m}$ of funding in the center is partitioned into projects and distributed to participating research groups in accordance to communal discussions between members of the center.

There are two benefits to this disbursement compared to grants originating directly from federal science agencies such as the NSF, DOE, and DOD. Firstly, bigger projects involving larger sums of money can be constructed amongst member research groups compared to the smaller grants awarded by federal agencies, which typically involve 1-3 principal investigators. Secondly, the overall portfolio of projects funded by the center must necessarily form a consistent picture which generates progress on the specific QIS challenge that the center focuses on. In the case of the QSA, this challenge is the scaling of quantum hardware platforms and the co-design of algorithms for scientific applications. Such a focus inevitably generates a project portfolio that is both more targeted and more interdisciplinary than those of federal science agencies, which are typically organized around individual disciplines in the physical sciences, life sciences, and engineering. Many of the salient challenges in QIS require a 'connected science' model to solve, where progress arises from a continuous exchange between fundamental science and engineering and at the intersection of different disciplines. The existence of centers like the QSA which can support a portfolio of variably sized projects that are thematically focused in a connected science approach is thus highly beneficial to progress in QIS.

The research funding funneled through the centers like the QSA supports graduate students at universities, scientific staff at national labs, and research technicians, thereby directly contributing to QIS workforce training and development. However, the center has not put forth concrete plans which sufficiently leverage its unique structure, that of a network 
of universities and national labs connected to an industry consortium, to most effectively grow the adaptable scientific workforce at the heart of a resilient and flexible QIS innovation system.

Scientists and engineers develop adaptability by regularly interfacing with colleagues from different lanes. For example, researchers at universities and national labs collaborating regularly develop an appreciation for the capabilities and career paths in each type of institution, while conversations between researchers and industry firms can grant the former a high level perspective on which applications have market demand and the latter a low level perspective on how mature the technology is and where the scientific challenges lie [18]. A workforce that has been trained to have these different perspectives will be more fluid, moving more easily between different actors in the innovation ecosystem and thus facilitating the diffusion of ideas and technical advances. Furthermore, interfacing with members of different research institutions and industry firms lets scientists and engineers develop an intuition for the goals and capabilities of different actors, increasing the likelihood of communication and collaboration between them. This communication and collaboration is a critical part of the resiliency and flexibility of the overall innovation system.

In the QSA, routine conversations between people working in different research areas and institutions, as well as between researchers and industry firms, seem to primarily occur between principal investigators and lab heads that form the decision body of the center. The structure of the center, however, provides a great opportunity for developing adaptability in the workforce by encouraging cross exposure between the scientists and engineers carrying out the research at different institutions, and between these researchers and industry firms.

As an example of the possibilities to exploit the synergies of the center, one can look to the local ecosystem set-up by two of its members: MIT and Lincoln Laboratory. Principal investigators working on engineering better superconducting circuit and ion trap platforms for QIS applications have created a 'one-team' model bridging researchers at both institutions. Graduate students at the MIT main campus spend more of their time on fundamental science and engineering challenges while staff researchers and technicians at Lincoln Laboratory spend more of their time on engineering and fabrication challenges, leveraging the relative strengths of the two locations. However, researchers at both places routinely interface and collaborate with each other as if they were part of a single unified research group. Additionally, MIT has its own consortium of industry firms interested in QIS called the Quantum Science and Engineering Consortium (QSEC). Researchers at both MIT and Lincoln are encouraged to have conversations with firms in QSEC, which provides the researchers with a perspective on industry needs while helping industry firms manage their expectations about the maturity and challenges of emerging quantum technology. This collaborative environment between a university, a national lab, and industry allows the scientists and engineers at MIT and Lincoln Laboratory to develop an adaptability that will make them invaluable parts of the QIS innovation system as they progress through their career.

Much of the success of the MIT-Lincoln environment stems from their geographic proximity and the long history connecting the two institutions. Themes underlying the success of this environment should, however, be extracted and applied where possible at the larger scale of the geographically decentralized QSA and other similar centers which are spiritually similar in their structure; just like the MIT-Lincoln environment, these centers provide a scaffold for collaborations between universities and national labs while maintaining a connection to an industry consortium. For example, when disbursing the center's funding into different projects, collaborations between researchers at universities and national labs may be encouraged when sensible. Additionally, a small component of the evaluation of a project's success could be to require researchers participating in the project to present their results to researchers from other groups in the center who were not part of the project. To encourage communication between researchers and industry, the center could find a relevant industry partner for each funded project through QED-C. The industry partner would not be required to fund any part of the research, but both the firm and researchers could have periodic conversations that provide context to each side. These relatively minor policies, and others like them, would allow the QSA and similar centers to catalyze the growth of an adaptable QIS workforce.

In summary, centers like the QSA provide a unique opportunity to coordinate the activity of various research groups within a common vision and thereby progress long-term goals necessary to advance QIS. However, more granular collaboration amongst members of the center and targeted policies fostering collaboration and cross-pollination are necessary to train the emerging QIS workforce. In particular, collaboration will be essential in training the new workforce to be adaptable enough to facilitate advances of these same goals in the larger national QIS ecosystem.

\section{Conclusions}

The NSF and DOE research centers instigated by the NQI provide a unique opportunity to develop adaptable scientists and engineers, who are crucial to the formation of a resilient and flexible QIS innovation system. However, researchers specialized in QIS are just one part of the workforce needed for this innovation system, and the recommendations we have listed here should be understood in the broader context of continuing government efforts to improve scientific workforce training. Large, diffuse ecosystems often face issues training and retaining talent, and the presence of similar challenges affecting the entire American scientific enterprise may be inferred from the prevalence of proposed legislation enacting improved mentoring programs (1)-(3), harassment prevention (4), and studying the mental health of students and researchers (5). 
Furthermore, policy approaches are only part of the solution to these issues. A strong scientific ecosystem relies on a collaborative culture, and where the culture of work is involved, a soft-power approach is called for. Promoting individuals cognizant of the structural challenges facing their workforce and promoting a culture conducive to a growing, healthy, and diverse workforce will be essential for the growth of QIS.

We are witnessing the birth of an industry, and the first truly quantum one at that. The beginnings of any industry are necessarily chaotic, and the current morass of companies, national laboratories, NSF joint centers, DOE centers, and university labs reflect a robust start for the growing QIS economy. The key to sustaining this growth will be developing a talented and multidisciplinary workforce.

\section{Acknowledgements}

We would like to acknowledge useful conversations with Will Oliver, Patrick Lee, William Bonvillian, Douglas Natelson, and William Brinkman. This work is partially supported by the NSF Graduate Research Fellowship under Grant No. 1745302.

\section{Citation}

Seetharam, K. \& and DeMarco, M. Catalyzing the quantum leap. MIT Science Policy Review 2, 26-30 (2021). https: //doi.org/10.38105/spr. lcbqc1igt5.

\section{Open Access}

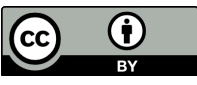

This MIT Science Policy Review article is licensed under a Creative Commons Attribution 4.0 International License, which permits use, sharing, adaptation, distribution and reproduction in any medium or format, as long as you give appropriate credit to the original author(s) and the source, provide a link to the Creative Commons license, and indicate if changes were made. The images or other third party material in this article are included in the article's Creative Commons license, unless indicated otherwise in a credit line to the material. If material is not included in the article's Creative Commons license and your intended use is not permitted by statutory regulation or exceeds the permitted use, you will need to obtain permission directly from the copyright holder. To view a copy of this license, visit http://creativecommons.org/licenses/ by $/ 4.0 /$.

\section{Legislation Cited}

(1) U.S. Congress. H.R.4483 (113th) STEM Mentoring and Inspiration Act of 2014. Introduced into Congress.

(2) U.S. Congress. H.R.5509/S.3583 (115th) Innovations in Mentoring, Training, and Apprenticeships Act. Passed by Congress.

(3) U.S. Congress. H.R.1665/S.737 (116th) Building Blocks of STEM Act. Introduced into Congress.

(4) U.S. Congress. H.R.36 (116th) Combating Sexual Harassment in Science Act of 2019. Introduced into Congress.

(5) U.S. Congress. H.R.1109/S.1122 (116th) Mental Health Services for Students Act of 2020. Introduced into Congress.

\section{References}

[1] Nielsen, M. A. \& Chuang, I. L. Quantum Computation and Quantum Information: 10th Anniversary Edition (Cambridge
University Press, USA, 2011), 10th edn.

[2] Ladd, T. D. et al. Quantum computers. Nature 464, 45-53 (2010). https: //doi.org/10.1038/nature08812.

[3] Degen, C. L., Reinhard, F. \& Cappellaro, P. Quantum sensing. Rev. Mod. Phys. 89, 035002 (2017). https://doi.org/10. 1103/RevModPhys.89.035002.

[4] Arun, G. \& Mishra, V. A review on quantum computing and communication. In 2014 2nd International Conference on Emerging Technology Trends in Electronics, Communication and Networking, 1-5 (2014). https://doi.org/10.1109/ ET2ECN.2014.7044953.

[5] Georgescu, I. M., Ashhab, S. \& Nori, F. Quantum simulation Rev. Mod. Phys. 86, 153-185 (2014). https: //doi.org/10. 1103/RevModPhys.86.153.

[6] Preskill, J. Quantum Computing in the NISQ era and beyond. Quantum 2, 79 (2018). https://doi.org/10.22331/q2018-08-06-79.

[7] Reiher, M., Wiebe, N., Svore, K. M., Wecker, D. \& Troyer, M. Elucidating reaction mechanisms on quantum computers. PNAS 114, 7555-7560 (2017). https://doi.org/10.1073/pnas. 1619152114.

[8] Bauer, B., Bravi, S., Motta, M. \& Chan, G. K.-L. Enabling quantum leap: Quantum algorithms for quantum chemistry and materials. Tech. Rep., National Science Foundation (2019). Online: https://www.nsf.gov/mps/che/workshops/ quantum_algorithms_for_chemistry_and_materials_ report_01_21-24_2019.pdf.

[9] Boerner, L. K. Industrial ammonia production emits more co2 than any other chemical-making reaction. (2019). URL https://cen.acs.org/environment/greenchemistry/Industrial-ammonia-production-emitsC02/97/i24. Accessed: July 2021.

[10] Smith, C., Hill, A. K. \& Torrente-Murciano, L. Current and future role of haber-bosch ammonia in a carbon-free energy landscape. Energy Environ. Sci. 13, 331-344 (2020). http: //doi.org/10.1039/C9EE02873K.

[11] Degen, C. L., Reinhard, F. \& Cappellaro, P. Quantum sensing. Rev. Mod. Phys. 89, 035002 (2017). https://doi.org/10. 1103 /RevModPhys. 89.035002.

[12] Wehner, S., Elkouss, D. \& Hanson, R. Quantum internet: A vision for the road ahead. Science 362, eaam9288 (2018). https: //doi.org/10.1126/science.aam9288.

[13] Ruess, F. The next decade in quantum computing-and how to play (2018). Online: https://www.bcg.com/frfr/publications/2018/next-decade-quantumcomputing-how-play. Accessed: February 2021.

[14] National Quantum Coordination Office. Quantum Frontiers Report. Executive Publication.

[15] Kjaergaard, M. et al. Superconducting qubits: Current state of play. Ann. Rev. Condens. Matter Phys. 11, 369-395 (2020). https://doi.org/10.1146/annurevconmatphys-031119-050605.

[16] U.S. Congress. H.R. 6227/S.3143 (115th) National Quantum Initiative Act. Passed by Congress.

[17] National Quantum Coordination Office. National strategic overview for quantum information science. Executive Publication.

[18] Aiello, C. D. et al. Achieving a quantum smart workforce. Quantum Sci. Techol. 6, 030501 (2021). https://doi.org/ $10.1088 / 2058-9565 / a b f a 64$. 